STUDI

FRANCESI

\section{Studi Francesi}

Rivista quadrimestrale fondata da Franco Simone

170 (LVII | II) | 2013

Varia

\title{
Les rondeaux et autres formes à refrain dans la "Moralité de Fortune et Povreté"
}

\section{G. Matteo Roccati}

\section{(2) OpenEdition}

1 Journals

\section{Édition électronique}

URL : https://journals.openedition.org/studifrancesi/2956

DOI : 10.4000/studifrancesi.2956

ISSN : 2427-5856

Éditeur

Rosenberg \& Sellier

\section{Édition imprimée}

Date de publication : 1 juillet 2013

Pagination : 347-361

ISSN : 0039-2944

\section{Référence électronique}

G. Matteo Roccati, «Les rondeaux et autres formes à refrain dans la "Moralité de Fortune et Povreté" », Studi Francesi [En ligne], 170 (LVII | II) | 2013, mis en ligne le 30 novembre 2015, consulté le 02 février 2023. URL : http://journals.openedition.org/studifrancesi/2956 ; DOI : https://doi.org/10.4000/ studifrancesi.2956

\section{(c) (i) $\odot$}

Creative Commons - Attribution - Pas d'Utilisation Commerciale - Pas de Modification 4.0 International - CC BY-NC-ND 4.0

https://creativecommons.org/licenses/by-nc-nd/4.0/ 


\title{
Les rondeaux et autres formes à refrain dans la "Moralité de Fortune et Povreté"
}

\begin{abstract}
The Moralité de Fortune, Maleur, Eur, Povreté, Franc Arbitre et Destinee (third quarter of the fifteenth century) is unpublished; as well as two ballads, it contains about ten texts in the forms "à refrain": "rondeaux", "fatras", "reffrain branlant". In some other passages it is possible to highlight anomalies in versification, anomalies that probably refer to particular forms that have been lost in the transition from the stage to the written record. The article provides editions of the texts and metrical analyses.
\end{abstract}

La Moralité de Fortune, Maleur, Eur, Povreté, Franc Arbitre et Destinee (terzo quarto del Xv secolo) è inedita; al suo interno, oltre a due ballate, una decina di testi sono delle forme "à refrain": "rondeaux", "fatras", "reffrain branlant". In alcuni altri passi si possono mettere in evidenza delle anomalie nella versificazione, anomalie che rinviano probabilmente a forme particolari che si sono perdute nel passaggio dalla scena alla traccia scritta. L'articolo fornisce l'edizione dei testi e ne analizza l'aspetto metrico.

La Moralité de Fortune et Povreté est un texte anonyme, composé sans doute dans le troisième quart $\mathrm{du} \mathrm{Xv}^{\mathrm{e}}$ siècle ${ }^{1}$. Il constitue la version dramatisée, enrichie de plusieurs personnages et de nombreux développements - philosophiques, juridiques, mythologiques, bibliques, historiques - du célèbre apologue raconté par Andalò del Negro en ouverture du livre III du De casibus de Boccace 2 .

La pièce met en scène la rencontre, puis la lutte entre Fortune, accompagnée de ses enfants, Maleur et Eur, et Povreté, accompagnée de Franc Arbitre, auxquels s'oppose également Destinee. La lutte et son issue - Maleur est attaché à un poteau qu'il pourra quitter uniquement pour suivre celui qui l'aura délié - est précédée et suivie de longues discussions entre les différents personnages. La métrique du texte est recherchée ${ }^{3}$ et quelques formes dites «fixes» sont intégrées à des moments particuliers de l'action.

(1) Cf. G.M. Roccati, "La Moralité de Fortune, Maleur, Eur, Povreté, Franc Arbitre et Destinee". Une adaptation $d u$ "Certamen paupertatis et fortune» (Boccace, "De casibus", livre III), in «L'analisi linguistica e letteraria», VIII (2000), 1-2, pp. 355 382. Pour une présentation du texte, en attendant l'édition, voir aussi maintenant ID., Du récit au dialogue, du latin au français: du "Certamen paupertatis et fortune» (Boccace, "De casibus", livre III) à la "Moralité de Fortune et Povreté", communication présentée au colloque Da un genere all'altro, To- rino, novembre 2010, maintenant dans Da un genere all'altro. Trasposizioni e scritture nella letteratura francese, a cura di D. Dalla Valle, L. Rescia, M. PAVESIO, Roma, Aracne, 2012, pp. 47-67.

(2) Cf. G.M. Roccati, "La Moralité de Fortune", cit., p. 376; Tutte le opere di Giovanni Boccaccio, a cura di V. BRANCA, vol. IX: De casibus virorum illustrium, a cura di P.G. RICCI e V. ZACCARIA, Milano, 1983, pp. XXIX-XXX, 940.

(3) Cf. G.M. RocCATI, "La Moralité de Fortune", cit., pp. 369-376. 
Deux ballades marquent l'entrée en scène de Povreté et la fin du texte ${ }^{4}$, une dizaine d'autres pièces à refrain sont reconnaissables à son intérieur, même si, à la différence des ballades, il n'y a pas de coupure marquée par rapport au contexte. Sauf dans un cas, il n'y a pas de changement de mètre et, comme cela se vérifie souvent pour les rondeaux, ces derniers sont parfaitement intégrés dans le continuum dramatique. Quatre de ces pièces sont des rondeaux réguliers ${ }^{5}$, deux autres des formes moins courantes, mais répertoriées dans la tradition; d'autres enfin sont ou des rondeaux imparfaits ou des formes difficiles à saisir, mais dans lesquelles un refrain apparaît ou des jeux de rimes curieux qui méritent qu'on s'y arrête.

Voici les vers en question, reproduits dans leur contexte, de manière à pouvoir se rendre compte de leur insertion, classés en fonction du schéma des rimes et numérotés, pour faciliter les renvois; tous sont en octosyllabes, à l'exception du $n^{\circ} \mathrm{V}$, en heptasyllabes 6 .

Textes I-IV

Rondeaux simples (triolets): $A B a A a b A B$

Rondeau I (vv. 486-493)

\section{Eur}

C'est pour donner a vos amys, tousjours leur estes vous courtoise.

\section{Maleur}

A saint sang Dieu! Qu'elle poise!

485

(a)

A

B
(4) Cf. G.M. Roccati, Les ballades insérées dans la "Moralité de Fortune et Povreté", in «Studi francesi», XLIX, n' 146, 2005, pp. 353-356.

(5) Sur le rondeau voir J. Cerquiglini, IV. Le rondeau, in Grundriss der romanischen Literaturen des Mittelalters (G.R.L.M.A.), dir. H.R. JAuss, vol. VIII, D. POIRION, dir., La littérature française aux XIV et XV siècles, t. I, Heidelberg, 1988, pp. 45 58, en particulier pp. 48-50. Pour son utilisation dans les textes dramatiques: T. KurorwA, «Le viel jeu» en mouvement: la configuration rimique et métrique des triolets dans les manuscrits du "Mystère de la Passion" d'Arnoul Gréban (La «Creacion du monde» et la première journée), in Vers une poétique du discours dramatique au Moyen Âge. Actes du colloque international organisé au Palais Neptune de Toulon les 13 et 14 novembre 2008, Textes réunis par X. Leroux, Paris, Honoré Champion, 2011, pp. 143-157; T. Kuroiwa, X. Leroux, D. Smith, De l'oral à l'oral: réflexions sur la transmission écrite des textes dramatiques au Moyen Âge, in «Médiévales», 59, automne 2010, pp. 17-40, en particulier pp. 33-37. Mes remerciements à Darwin Smith et aux autres auteurs pour m'avoir communiqué ces articles avant parution.

(6) La majuscule indique le refrain (matérialisé par l'espacement étendu des caractères); entre pa- renthèses les rimes reliant les formes identifiées au contexte: il s'agit très souvent d'un vers adjoint qui assure la transition entre les couplets et le rondeau. Sur le «vers adjoint», cf. Taku Kuroiwa, Xavier LEROUx, Darwin SMITH, Formes fixes: futilités versificatoires ou système de pensée?, in Vers une poétique du discours dramatique, cit., pp. 121-142, en particulier pp. 141-142. L'intuition éclairante exposée dans cet article - le couple $a b$ (et non aa) considéré comme base de la versification dramatique médiévale - s'applique dans nos cas particuliers à l'intérieur des rondeaux, mais le lien de ces derniers avec le contexte se fait en général, et selon l'habitude, par un vers adjoint. La numérotation des vers est celle de l'édition critique du texte qui devrait bientôt paraître. Une première transcription se trouve dans S. LEONE, Una «moralité en vers» inedita del XV secolo (ms. Paris, B.N.F., n.a.f. 6218), tesi di laurea inédite (Università di Torino, Facoltà di lettere, a.a. 1996-97).

(7) Ms. Paris, B.n.F., n.a.f. 6218, f. 11r.

(8) ... Comme elle me pèse, je crois qu'elle m'anéantira: dans le jeu scénique Maleur devait vaciller, écrasé par le poids de sa malle.

(9) À la fois le mauvais sort et la malle de Fortune, dans laquelle il ne manquera jamais du bien (des «richesses»). 
de qui jamais bien ne fauldra.

Se garde de moy qui vouldra!

a

A

Eur

490 Je porteray, il le faudra,

la bonne a qui nul ne repugne ${ }^{10}$.

a

$\mathrm{b}$

\section{Maleur}

Se garde de moy qui vouldra, je porte la malle fortune, mais ce n'est point chose oportune,

495 ce m'est advis, ne bien honneste, de porter cecy sur ma teste.
A

B

(b)

Rondeaux II et III ${ }^{11}$ (vv. 2185-2192, 2194-2202)

\section{Maleur}

Si cray bien donc qu'il eslivra avec moy la malle sancté.

Povreté

2185 Maleur! C'est assés cacqueté, a ce pal vous fault atachier.

\section{Maleur}

Helas seray-je ainsi traictié?

Franc Arbitre

Maleur! C'est assés cacqueté.

Maleur

Est ce point grant iniquité

2190 de moy en ce point empescher?

a

b

\section{Povretét $^{12}$}

Maleur! C'est assés cacqueté, a ce pal vous fault atachier.

A

B

\section{Povreté}

L'en vous gardera de mon chier Franc Arbitre.

(10) Sur la rime fortune/repugne, cf. C. MARCHELlO-NIZIA, La langue française aux XIV et XV siècles, Paris, Nathan, 1997, p. 113.

(11) F. 39r-v.
(12) Rubrique sans doute à corriger en Franc Arbitre, pour éviter la répétition avec la rubrique suivante. 
Franc Arbitre

$$
\text { Quoy? }
$$

Povreté

$\begin{array}{ll}2195 \text { Liez bien! } & \text { C } \\ \text { Maleur } & \text { D } \\ \text { Helas! Faictes moy quelque bien } & \text { c } \\ \text { Franc Arbitre. }\end{array}$

Franc Arbitre

$$
\text { Quoy? }
$$

Povreté

$$
\text { Liez bien!// C }
$$

f. 39v Franc Arbitre

Le lacheray je ung pou.

\section{Poureté}

Rien, Rien!
J'aymeroye mieulx a estre morte,
2200 Franc Arbitre.

c

Franc Arbitre

$$
\text { Quoy? }
$$

Povreté

\[ \text { Liez bien! } \]
Il est de tres malvaise sorte!
Quelle chere faictes vous! [Hay!]

C Quelle chere faictes vous! [Hay! $]^{13}$

Maleur

Morte! (d)

Povreté

Se vous vous savez despeschier pour bien parler ne pour prescher

2205 vous serés nigromancien.

(13) Le vers compte neuf syllabes, cette exclamation a sans doute été introduite par inattention.

(14) F. 39v-40r. 
Rondeau IV ${ }^{14}$ (vv. 2218-2226)

\section{Povreté}

Et puis ceste malle jolie

2215 l'attacherons nous point icy?

Franc Arbitre

J'en suis content.

Povreté

Et moy aussi.

Je n'en ayme point la couleur.

(a)

Franc Arbitre //

f. 40r Celuy qui desliera Maleur la malle fortune prendra.

Povreté

2220 Il multipliera sa douleur celuy qui desliera Maleur.

Franc Arbitre

A paine aura jamaiz honneur qui sur son col la chargera.

a

\section{Povreté}

2225 Celuy qui desliera Maleur la malle fortune prendra.

\section{Fortune}

Qui me dira quant ce sera (b) que ma grant douleur cessera, qui est tant gresve et tant amere, car je ne sçay quant fin aura,

2230 ou se la vie finira de la tres douloureuse mere.

A

$\mathrm{b}$

B

Texte $\mathrm{V}^{15}$ (vv. 697-709)

Fatras (epilogue): $A B A a b a a b b a b a B$ Heptasyllabes

$$
\begin{aligned}
& \text { Povreté } \\
& \text { J'ay ma maisonnete } \\
& \text { bien gentement faicte, } \\
& \text { par dedens joyeuse et necte, }
\end{aligned}
$$

(15) F. 15r-v. 
685

690

695

f. $15 \mathrm{v}$

700

705

710 Maiz qui est ceste damoiselle qui ci en l'oreille nous chante et est tant povre et tant meschante que j'ay grant orreur de la voir? ou sont mes enfans.

Doulcement les traicte

et les admoneste

d'estre a Dieu, par voye honneste,

vrais obeyssans.

Beaulx dictiers plaisans

et gracieulx champs ${ }^{16}$

font aux gardins et aux champs

soubz une espinete.

La sont en tout temps

lyés et esbatans,

on ne trouveroit en cent ans

vie plus doulcete.

Mes gens sont tousjours joyeulx. A

Est-il telle vie, telle? // B

Mes gens sont tousjours joyeulx, A

de rien ne sont curieulx, a

fors de la vie eternelle. b

Depuis l'eure que vis eulx a

je ne les vis envieulx a

sur creature mortelle. b

Dame Paix la bonne et belle b

est tousjours avecques eulx a

qui mainte chansson nouvelle b

leur fait dire deulx et deulx: a

est-il telle vie, telle? B

$A$
$B$
$A$
$a$
$b$
$a$
$a$
$b$
$b$
a
a
$B$


qu'autant luy soit des morfondus, et des res, comme des tondus ${ }^{19}$.

b

Son parler m'estonne et affolle.

b

c

\section{Fortune}

720 Passons oultre, c'est une folle.

\section{Povreté}

C'est une folle, ton langaige.

Ce monstre que tu n'ez pas saige:

de fol juge sentence bresve.

Se je di chose qui te gresve,

argue contre ma parolle. //

d
d
e
e
$c$

C

Passons oultre, c'est une folle.

\section{Povreté}

Voire toy, selon mon advis.

Bien sçay qu'onques mais ne me vis.

Or, puis que tu ne me vis oncques,

comment juges tu de moy doncques, ai-ge dit mensonge ou frivolle?

\section{Fortune}

Passons oultre, c'est une folle.

Il ne s'i fault ja arrester,

elle se peult assez vanter,

.

C

Ces six premiers textes, et notamment les rondeaux, sont tout à fait conformes à la tradition, dans leur schéma comme dans leur situation: ils interviennent à des moments clé de l'action ${ }^{21}$. Le rondeau I est inséré à la fin de la première partie introductive où Fortune apparaît avec ses enfants. Le dialogue entre ces deux derniers fait suite à l'exhortation de leur mère pour qu'ils investissent le monde pour rétablir sa puissance. Maleur se met en route, décidé à porter aux hommes la malle de sa mère (vv. 479, 809, 2214), contenant la malle fortune (vv. 487, 493, 2219, 2226), et Eur, de son coté, s'apprête à porter la bonne. Les rondeaux II et III s'enchainent l'un à la suite de l'autre au moment, de grande agitation, où Franc Arbitre, à la demande de

(19) Il semble qu'ils lui soient pareils les malades, les rasés et les tondus (les fous). Cf. Meschin., Lun. princes, 13: «Je donne autant des rez que des tondus» (DMF 2012).

(20) Cf. Thesaurus proverbiorum medii aevi, Begründet von S. SINGER, Hrsg. von Kuratorium Singer der Schweizerischen Akademie der Geistesund Sozialwissenschaften, Berlin, New York, W. de Gruyter, 1996-2002 (TPMA), s.v. «Richten», nos 65-70 (IX, p. 294); G. Di Stefano, Dictionnaire des locutions en moyen français, Montréal, Éd. CERES,
1991, p. 463c.

(21) Sur le rondeau comme «indicateur conventionnel de simultanéité», «code d'organisation vocale destiné à reproduire l'enchevêtrement verbal d'une situation collective», voir T. KUROIWA, X. LERoux, D. Smith, De l'oral à l'oral, cit., p. 29. Dans notre cas il s'agit surtout de signifier un moment clé: à part le moment où Maleur est attaché au poteau, il ne s'agit pas nécessairement d'«enchevêtrement», ce dernier est plutôt réalisé par le fractionnement des vers. 
Povreté, attache Maleur au poteau, qui, de son coté, se plaint de ce qu'on lui fait. Cette agitation est traduite par le fractionnement des reparties, jusqu'à trois dans un octosyllabe. Le rondeau IV intervient peu de vers après les deux autres, lorsque Povreté, après avoir accroché la malle de Fortune, dialogue avec Franc Arbitre en concluant la scène, avant que Fortune intervienne à son tour dans sa lamentation finale.

On voit bien que ces pièces, comme c'était le cas des ballades, interviennent à des tournants dans l'action dramatique: l'utilisation d'une forme «fixe», avec le jeu de scène qui devait aller avec, sert à en souligner l'importance. Les rondeaux I, II, III, IV, tous en octosyllabes, ont la forme traditionnelle du triolet $(A B a A a b A B)$. Selon une technique tout à fait habituelle, le premier et le dernier vers forment couplet avec les vers précédant et suivant ${ }^{22}$, reliant entre elles les reparties ${ }^{23}$. D'un point de vue dramatique on peut souligner le jeu entre forme métrique et répliques. Dans le rondeau I les quatre premiers vers constituent l'essentiel de la réplique de Maleur, martelée par le refrain, entrecoupée par les deux vers autonomes, mis dans la bouche de Eur. Dans les rondeaux II et III, avec de petites variations, le refrain constitue le nerf de l'intervention de Povreté, soutenue grâce au vers isolé du refrain par Franc Arbitre, les deux s'opposant à Maleur. Dans le rondeau IV Franc Arbitre et Povreté jouent une sorte de partition à deux voix à propos de la malle fortune.

À coté de ces rondeaux, on relève deux autres formes à refrain. Le texte $\mathrm{V}$, le seul en heptasyllabes, est un fatras ${ }^{24}$. Le schéma $\left(A B A a b a a b b a b a B^{25}\right)$, même s'il est moins fréquent que celui du rondeau, est bien connu: la structure est décrite par Pierre Fabri, il la nomme «epilogue», et il ajoute «que les Piccars [l'] appellent en leur langage fatras; et se faict de xi. lignes communement, mais on les peult faire plus ou moins, nom per, courtes ou longues, et tant de clauses que toute la premiere clause soit epilogue; et conuient reprendre la premiere ligne pour tierce ligne, et la seconde au dernier ${ }^{26}$. Ce fatras intervient juste avant que Fortune s'aperçoive de la présence de Povreté qui est arrivée sur scène en récitant une ballade et continue ensuite en chantant, tout en dialoguant avec Franc Arbitre; il conclut la séquence. Les treize heptasyllabes du fatras font suite à une structure plus complexe en heptasyllabes et pentasyllabes (vv. 681-696: $2 \times a a^{5} a^{7} b^{5}+2 \times b^{5} b^{7} a^{5}$ ) et terminent la repartie. La repartie suivante, où Fortune intervient et dont le premier vers rime avec le dernier de la repartie précédente, est en octosyllabes.

Tout de suite après ce fatras, les vers du texte VI - schéma aabbcC ddeecC ffggcC, à noter que le refrain constitue à chaque fois la repartie de Fortune - correspondent bien à la définition du «reffrain branlant» que donne Pierre Fabri: «Reffrain branlant ou vollant se faict, quant les lignes se croysent en rithme de vne mesme ou diuerse mesure; mais la derniere est en reffrain, et faut du moins quattre lignes en clause» ${ }^{27}$.

(22) Sauf le premier vers du rondeau III (v. 2194), orphelin: il ne s'agit pas d'un oubli dans la transcription, les vv. 2193 et 2194 s'enchainent logiquement. Un problème dans la copie n'est pas toutefois à exclure, comme le montre la répétition de la rubrique aux vv. 2191 et 2193.

(23) Il s'agit de la rime dite «mnémonique», à ce sujet, cf. G.A. RunNalls, Monologues, dialogues et versification dans le «Mystère de Judith et Holofernes», in L'économie du dialogue dans l'ancien théâtre européen, Actes de la première rencontre sur l'ancien théâtre européen de 1995, réunis par J.-P. Bordier, Paris, Champion, 1999, pp. 115-139, en particulier pp. 119-121.

(24) Cf. H. Chatelain, Recherches sur le vers français au $X V^{e}$ siècle. Rimes, mètres et strophes, $\mathrm{Pa}$ - ris, 1907, reprint Genève, 1974, pp. 222-224.

(25) La segmentation du schéma entend simplement mettre en lumière la structure: tous les vers appartiennent à la même intervention de Povreté et sont nettement identifiés comme un tout par leur homogénéité métrique

(26) P. FABRI, Le Grand et Vrai Art de pleine rhétorique, publié (...) par A. Heron, Rouen, 1889-1890, t. 2, p. 82; ici le schéma est dans sa forme la plus simple, il peut y avoir plusieurs «clauses» avec une reprise complexe des vers: description et exemples de cette «espece ou maniere de rithmer» aux pp. 82-85.

(27) P. FABRI, Le Grand et Vrai Art de pleine rbétorique, cit., t. 2, p. 85, cité par T. KunOIWA, «Le viel jeu» en mouvement, cit., p. 147, qui traite de cette forme dans les arts rhétoriques. 
La différence par rapport à la ballade est dans les rimes, qui ne sont pas les mêmes, et dans le nombre de «clauses»: «autant que l'en veult» ${ }^{28}$.

$$
* * *
$$

À ces formes, plus ou moins courantes, mais tout à fait conformes à celles répertoriées, on peut ajouter quelques autres vers qui présentent des particularités les rattachant et les distinguant à la fois des schémas habituels.

Texte VII ${ }^{29}$ (vv. 1409-1415)

Rondeau imparfait: $A B a A a b c$

\section{Maleur}

Or pendre puist on d'une corde qui ne fera mieulx qui pourra.

Povreté

Je ne sçay qu'il en adviendra, maiz je l'assauldray la premiere.

Eur

Pense qu'on te recevra,

je ne sçay qu'il en adviendra.

\section{Franc Arbitre}

Avant Povreté! On verra se vous estes forte et entiere.

Poureté

1415 Je ne sçay,

mais je l'assauldray.

\section{Eur}

Povreté est si tres legiere a

A

a

B

b

que riens a son cas ne repugne.

Ces vers, où s'expriment les dernières exclamations belliqueuses qui précèdent la lutte, constituent de toute évidence un rondeau, mais le v. 1415, le dernier, est coupé en 3+5 syllabes dans la copie même, les deux parties rimant entre elles. Il s'agit en fait du début des deux vers du refrain du rondeau et il faut noter que le v. 1416, dont la rime est orpheline, devrait en fait rimer, comme «vers adjoint» avec le deuxième vers du refrain. On ne peut donc pas exclure que le rondeau soit tout à fait régulier et la lacune due au copiste, le début des deux vers étant écrit pour mémoire et devant être complété lors de la performance. Cependant le fait que le v. 1415 soit un octosyllabe, avec rime interne, me semble montrer que la suppression du refrain

(28) «et faict l'en six ou huyt clauses de refrain vollant, ou autant que l'en veult, et tout de diuerses lisieres, rien gardé que le reffrain» (P. FABRI, Le
Grand et Vrai Art, ibidem).

(29) F. 27v. 
est voulue, pour terminer de manière lapidaire les préalables de la lutte et sans doute aussi pour surprendre l'auditoire, qui s'attendait au refrain; on serait là dans une sorte de deuxième degré par rapport à la forme habituelle.

Texte VIII ${ }^{30}$ (vv. 1419-1430)

Franc Arbitre

La! Povreté!

Maleur

Avant Fortune!

Faictez que vous ayez victoire ${ }^{31}$ !

a

\section{Fortune}

1420 Te rens tu?

Povreté

Nenni, pas encore!

a

Eur

Fortune! Donnez luy ung sault!

B

Fortune

Crie mercy!

Povreté

Il n'en est memore.

a

Franc Arbitre

Povreté! Donnez luy ung sault!

B

Fortune

Helas Maleur! Le cueur me fault.

$\mathrm{b}$

Maleur

1425 Mourrez vous en ceste bataille? //

C

f. $28 \mathrm{r} \quad$ Fortune

Ceste Povreté coupe et taille

C mes rains.

Eur

Gardez qu'el ne vous serre.

d

(30) Ff. 27v-28r.

(31) Sur la graphie -oire / -ore, cf. Ch. MARCHELLO-NIZIA, La langue française, cit., p. 81; Le Mystère de la Passion d'Arnoul Gréban, éd. critique par O. Jodogne, t. II, Bruxelles, 1983, p. 40. 


\section{Fortune}

Je n'ay membre qui me faille.

Franc Arbitre

Bon, vela Fortune par terre.

Povreté

1430 Vous vous sentirés de la guerre, vous avez huy trop quaqueté.

\section{Fortune}

Pour Dieu! Ostés moy Povreté qui tient contre moy ses argus. Ses genoulx sont si tres agus

1435 qu'ilz me percent tous les costés.

\section{Franc Arbitre}

Gallans! Se vous vous y boutés, tres grant mal vous en pourra prendre.

Povreté

Rens toy!

Fortune

Helas me fault il rendre!

Pour Dieu! Que vous nous separés!

Povreté

1440 A! Sur ma foy, vous vous rendrés tantost puis que je l'entreprens.

\section{Fortune}

Helas Povreté! Je me rens.

Mect telle loy que tu vouldras.

Povreté

Tu jures que tu le tendras

1445 sans point desobeir a moy?

Faiz pas!

Fortune

Ce faiz mon ${ }^{32}$, par ma foy! C'est raison que je m'y consente. 


\section{Povreté}

Or te lieve, je suis contente,

mais que tu tiennes ta promesse!

Cette séquence très animée correspond au moment de la lutte, elle s'enchaîne directement après notre texte VII. Le schéma des rimes des vv. 1419-1430 est bizarre: $a a B a B b c c d c d d^{33}$. Même si le refrain en réalité n'en est pas complètement un, car le début change - les vers commencent par Fortune ou Povreté, le reste du vers est identique, Donnez luy ung sault -, il est probable que dans les vv. 1420-1424-aB $a B b$ - reste la trace d'un refrain récité sur scène dans le cadre d'une forme qui n'a pas été reprise dans la copie ${ }^{34}$.

La construction du v. 1438: «Rens toy! / Helas me fault il rendre!», analogue à la structure des vv. 1420 et 1422, comme le v. 1431, à rapprocher d'un vers du refrain du rondeau II (vv. 2185, 2188, 2191), sont peut-être d'autres indices d'un échange organisé comme un rondeau. La situation en tout cas s'y prêterait bien.

Texte IX $^{35}$ (vv. 1370-1380)

\section{Fortune}

Qu'en dis tu Maleur, mon enfant, la combatrai-ge sur ce point?

\section{Maleur}

1370 Pour Dieu ne la refusez point! Du premier coup la ferez rendre.

A

Eur

Vous estes grasse, blanche et tendre, b

et si avés tousjours esté

c

tres bien nourrie, et Povreté

n'a sans plus que les povrez os.

c

1375 Vous la combatrez.

d

\section{Fortune}

$\begin{array}{ll}\quad \text { En briefs mos: } & \mathrm{d} \\ \text { faictes la moy venir en feste // } & \mathrm{e} \\ \text { se par moy luy est mercy faite } & \mathrm{e} \\ \text { sur ma foy je vueil qu'on me tonde }{ }^{36} \text {. } & \mathrm{f}\end{array}$

Esse bien dit?

Maleur

$\begin{array}{ll}\text { Le mieulx du monde. } & \mathrm{f} \\ 1380 \text { Pour Dieu, ne la redoubtez point! } & \text { A }\end{array}$

(33) J'introduis des blancs pour mieux faire ressortir le schéma, en réalité chaque repartie occupe au maximum deux vers, le plus souvent un seul ou un fragment de vers.

(34) L'exemple d'un rondeau «resorbé» dans une des copies qui nous restent se trouve dans le
Mystère de la Passion, cf. T. KurOIWA, «Le viel jeu» en mouvement, cit., pp. 148-149.

(35) Ff. 26v-27r.

(36) J'accepte d'etre tenue pour folle si... (DMF 2012; G. Di Stefano, Dictionnaire des locutions, cit., pp. 841c-842a). 


\section{Franc Arbitre}

Ça, vecy Povreté en point

qui ne quiert que ces advantures.

Nous sommes ici au moment des dernières considérations avant la lutte, à l'intérieur de la suite des couplets les vv. 1370 et 1380 - Pour Dieu ne la refusez / redoubtez point - ressemblent beaucoup à un refrain bien qu'ils soient parfaitement intégrés dans le continuum d'octosyllabes. Ici aussi la situation se prêterait bien à la présence d'un rondeau.

$$
* * *
$$

Enfin on peut encore citer deux textes où aucun refrain n'apparaît, mais où le schéma des rimes laisse entrevoir une structure plus complexe que celle apparente.

Texte $\mathrm{X}^{37}$ (vv. 165-180)

160 le tres grant couroult qui m'opresse.

Compagnie me fault expresse, car je desire

trouver a qui je me desyre

et qui me donne au cueur le<e $>$ sse:

165 Bon Heur.

Eur

Me vecy ma princesse se vous avés de moy beso $<\mathrm{i}>\mathrm{ng}$.

Fortune

Maleur?

\section{Maleur //}

f. $5 \mathrm{r}$

Que vous plaist ma maistresse?

a

\section{Fortune}

Ou es tu?

\section{Maleur}

Je ne suis pas loing.

\section{Fortune}

Mes enfans je suis en grant soing b

d'une chose que vous diray

car je vous promets que dire ay

plus qu'on ne sçauroit exprimer.

J'ay veu qu'on me souloit amer

b

et avoir en grant reverence: 


\section{Maleur}

Je cuide moy, comme je pence, que ce fust en l'aage doré.

Il s'agit ici du premier dialogue entre Fortune et ses enfants, l'enchainement des octosyllabes est bizarre: $a b a b b c c$ dde de ef ef. Le jeu des rimes se fait entre les reparties: $a b(E) a(F M) b(F M) b c c-e f(F)$ ef $(M)$. Ce schéma curieux cache-t-il la trace d'une forme particulière? La situation - le premier jeu à trois entre Fortune et ses enfants - pourrait bien justifier l'hypothèse.

Texte $\mathrm{XI}^{38}$ (vv. 932-936)

Ce roy qui oncques n'ot pareil se mist encontre son soleil

930 luy priant qu'il le peust avoir et qu'il luy donrroit grant avoir. Mais de sa responce l'a point, car il respondi sur ce point: a «Tes dons vueil tous habandonner, mais beau sire ne m'oste point ce que tu ne me peulx donner».

\section{Fortune //}

f. $20 \mathrm{r}$

Je ne faiz conte de mener telz meschans gens avecquez moy.

940 tu peulx congnoistre clerement que j'ay en mon gouvernement l'universelle monarchie.

Ce texte s'insère dans le débat où les deux femmes revendiquent chacune la prééminence sur l'autre. Sur une suggestion de Franc Arbitre, Povreté apporte comme preuve de la sienne l'anecdote célèbre de Diogène et Alexandre ${ }^{39}$. Le schéma des rimes est curieux: il pourrait s'agir d'une forme attestée par ailleurs ${ }^{40}$, mais la rupture du couplet et la reprise des mêmes mots à la rime pourrait aussi bien être la trace d'un jeu disparu lors de l'écriture. Il est bien évident toutefois qu'il n'est pas possible d'aller au delà de l'hypothèse.

(38) Ff. 19v-20r.

(39) Depuis Valère Maxime (Dict. mem., IV, 3 , ext. 4) elle est très répandue, parmi les œuvres approximativement contemporaines de la moralité, elle se trouve notamment chez Martin Le Franc dans L'Estrif de Fortune et Vertu (éd. crit. par P.F. Dembowski, Genève, Droz, 1999, p. 244; cf. aussi p. 333, n. 242; O. RотH, Studien zum "Estrif de Fortune et Vertu" des Martin Le Franc, Bern, 1970, pp. 197, 314-315).

(40) Notamment dans La condamnation de Banquet, vv. 1067-1071: aab ab; éd. J. KoOpmans et P. Verhuyck, Genève, Droz, 1991, cf. p. 49. Voir aussi les «formes courtes» traitées par Chatelain et Noomen, cf. W. NoOmen, Etude sur les formes métriques du Mystère du Vieil Testament, Amsterdam, 1962, pp. 68-82. 


$$
* * *
$$

Quelques mots, pour finir, sur ce que ces formes intégrées dans les reparties nous révèlent de la conception du texte dramatique de l'époque. Elles sont manifestement un élément constitutif de ce dernier ${ }^{41}$ et cette simple constatation permet d'apercevoir combien il est différent par rapport à nos habitudes. L'absence de mimétisme réaliste semble être à première vue ce qui nous le rend le plus lointain, mais la dimension métrique joue sans doute un rôle encore plus important dans son éloignement. Il nous est difficile d'avoir la même perception, de comprendre ce que ces échanges stylisés représentaient pour les acteurs et le public ${ }^{42}$. Nous touchons là à un aspect qui échappe à une lecture naïve car il ne fait plus partie de notre attente, seul le travail critique permet de le reconstituer ${ }^{43}$.

G. MATTEO ROCCATI

(41) Sur l'importance de cet aspect, cf. T. KuRoIwA, X. Leroux, D. Smith, Formes fixes, cit.

(42) S. ROBERT, Formes poétiques et versification dans "La sotise à buit personnages" d'André de la Vigne, in Langues, codes et conventions de l'ancien théatre, actes de la troisième rencontre sur l'ancien théâtre européen, Réunis par J.-P. BORDIER, Paris,
Honoré Champion, 2002, pp. 55-66, les interprète comme le fruit d'un jeu entre structure et désordre, ce qui peut être éclairant, mais ne suffit pas.

(43) Toute ma reconnaissance va à Darwin Smith qui m'a sensibilisé à cette dimension du texte, dimension qu'auparavant je ne voyais pas. 